\title{
Experiment on building a real-time temperature field distribution model of the prostate using special data encryption multi-pole radiofrequency ablation and a visualization phantom
}

\author{
CHEN Lei, HU Bing* \& HU Bin \\ Department of Ultrasound, Affiliated Sixth People's Hospital, Shanghai Jiaotong University, Shanghai 200233, China
}

Received February 28, 2011; accepted July 27, 2011

\begin{abstract}
The aim of this study was to explore the utility of a visualization phantom for building a real-time temperature field distribution model of the prostate created by the application of special data encryption (SDE) multi-pole radiofrequency (RF) ablation. We prepared phantoms (phantom group) using acrylamide as the raw material and egg-white as a color developing reagent. The prostate specimens of male adult dogs were fixed in this phantom to create a prostate phantom (prostate group). The SDE multi-pole RF electrode was inserted into the two groups for RF ablation, and the temperature changes at relevant points in space were detected. We set the $x$-axis as the left and right direction, the $y$-axis as the fore and aft direction, and the $z$-axis as the insertion direction of the RF electrode. After this process, the effect of RF ablation on the appearance of each of the two groups was observed. Using temperature measurements taken during the operation, a three-dimensional (3D) surface model of temperature was constructed by drawing the boundary areas of different temperatures on the maximum $x$ - $z$ plane of the two groups of ablation lesions, with $y$ equivalent to 0 . Further comparison of the temperature-time change curves of four space points were made by setting $y$ equivalent to $0, z$ equivalent to 0.5 , and $x$ equivalent to $0,0.25,0.5$ and 0.75 . White solid lesions with a diameter of approximately $12 \mathrm{~mm}$ could be found in both groups after RF ablation. During the ablation of the phantom group, the temperature ascended faster in a zone $1 \mathrm{~cm}$ from the needlepoint to the needle body, and $0.5 \mathrm{~cm}$ about the radiofrequency needle. The time-property result of the $x-z$ plane with $y$ equivalent to 0 showed that the boundary areas of the two groups were closer at 50 and $60^{\circ} \mathrm{C}$, but the difference between the two groups at $70^{\circ} \mathrm{C}$ in the high temperature zone was relatively large. The $3 \mathrm{D}$ temperature surface model further proved that the $3 \mathrm{D}$ temperature outline at $60^{\circ} \mathrm{C}$ was in better concordance with the actual effect of RF ablation in the prostate tissue. In the comparison of temperature-time changing curves of the two groups at the four space points, despite there being a significant difference in the changing process $(P=0.0001)$, there was no significant statistical difference in the final temperature of the two groups $(P>0.05)$. The results of this work indicated that ablation appearance was in good agreement with in-vitro biologic tissues and that the RF temperature field distribution can be obtained by using a visualization phantom. In particular, the shape of the isothermal line at $60^{\circ} \mathrm{C}$ was close to the actual ablation lesion. These results may serve as a valuable reference to future clinical trials.
\end{abstract}

visualization phantom, multi-pole radiofrequency ablation, prostate

Citation: $\quad$ Chen L, Hu B, Hu B. Experiment on building a real-time temperature field distribution model of the prostate using special data encryption multi-pole radiofrequency ablation and a visualization phantom. Chinese Sci Bull, 2011, 56: 3845-3853, doi: 10.1007/s11434-011-4777-4

In recent years, the incidence of prostatic carcinoma has been ascending in the most regions of the world. With the widespread use of digital examination of prostate through the rectum and prostate specific antigen screening, the ear-

*Corresponding author (email: hubing9901790@yahoo.com.cn) lier diagnosis rate of prostatic carcinoma has also been ascending along with disease incidence. Although radical prostatectomy is the first choice in treatment of such diseases, it is questionable whether senior patients with prostatic carcinoma or other serious basic diseases would be able to endure such surgery. By contrast, some partial treatment 
methods such as radiofrequency (RF) ablation treatment involve less trauma while having good effectiveness and consequently may be more appropriate for these patients [1]. According to the literature, RF ablation treatment for prostatic carcinoma by the insertion of a needle from the perineum is easy to perform and is convenient; the operation process is easy to monitor; the neoplastic metastatic rate is low and the incidence of associated complications is low. As a result, it has been advocated by many clinicians $[2,3]$.

In consideration of the fact that the prostate is of small volume and is surrounded by the rectum, bladder and seminal vesicles, with the urethra passing through the center, the main point of RF treatment against prostatic carcinoma lies in how to conduct a thorough ablation of the carcinoma while protecting the surrounding normal tissues. According to the latest research $[4,5]$, the answer to this problem is to ensure meticulous control over the RF parameters. The basis upon which this control is made feasible is derived from real-time monitoring and careful analysis of the temperature field distribution of the whole ablation area. In contrast with the method of obtaining temperature field distributions of thermal destruction treatment by a parsing algorithm with finite elements [6], the best clinical solution would be to develop a phantom with basic thermodynamic parameters that are closer to human body tissue, and then use this to intuitively observe the shape of the ablation lesion. Therefore, to analyze the real-time temperature field distribution in three dimensions, this research aims to build an in-vitro phantom of a prostate RF ablation lesion to aid visualization, by simulating the temperature changing process of real RF ablation against prostate tissue. Furthermore, this research aims to provide a valuable reference for the future design of reasonable ablation proposals for prostatic carcinoma patients, and to contribute to the development of next-stage clinical trials.

\section{Materials and methods}

Stock solutions of phantom were prepared in accordance with the following proportions: $44.5 \mathrm{~mL}$ degassed pure water
(Amersco, Solon, OH, USA), 20\% mL egg white, $24.8 \mathrm{~mL}$ acrylamide solution of $40 \%$ (Amersco), $0.5 \mathrm{~mL}$ AP of $10 \%$ (Amersco) and $0.2 \mathrm{~mL}$ TEMED (Amersco) in every $100 \mathrm{~mL}$ of stock solution.

For the in-vitro phantom group, the phantom stock solution was injected into a special organic glass box and was left for $20 \mathrm{~min}$, to make a phantom with a volume of $280 \mathrm{~mL}$; 390 phantoms were made this way in total. We then inserted the SDE multi-pole RF electrode into the appointed position of the phantom and carried out the relevant RF ablation operation according to preset parameters (Figure 1(a) and (b)). According to the position and size of the RF ablation lesion produced in the experiment, the edge was enlarged by 10 $\mathrm{mm}$ and a solid square matrix was drawn with $5 \mathrm{~mm}$ spaces in three dimensions. With the end point of the unexpanded $\mathrm{RF}$ electrode as the origin, we set the $x$-axis (the left-right direction of the expanding RF electrode), $y$-axis (for the fore-aft direction of the expanding RF electrode), and $z$-axis (for the insertion direction of the RF electrode, the origin point is the end point of the unexpanded RF electrode). We then measured the temperature change in the matrix at every designated point in space, one by one with an interval of $2.5 \mathrm{~mm}$, and repeated the measurement of the temperature change of each point three times. Before measuring the temperature, each probe was immersed into an ice-water mixture and warm water of $50-70^{\circ} \mathrm{C}$. The probe was covered with insulating paste so that the influence of the electric field could be reduced. For the in-vitro prostate phantom group, 49 male adult dogs were euthanized by air-injection, and the prostate specimens were excised $2 \mathrm{~h}$ post-euthanasia. The diameters of all the prostate specimens were required to be above $30 \mathrm{~mm}$. After washing the prostates, a 14G puncture guiding needle was inserted into the prostate specimen and placed in the above-mentioned stock solution. The guiding needle was then extracted until the stock solution was completely solid. The SDE multi-pole RF electrode was then inserted into the appointed position of the phantom, and the relevant RF ablation operation was carried out according to the preset parameters (Figure 1(c)). The temperature measurement was the same as before; however, it was only necessary to measure the temperature change at space
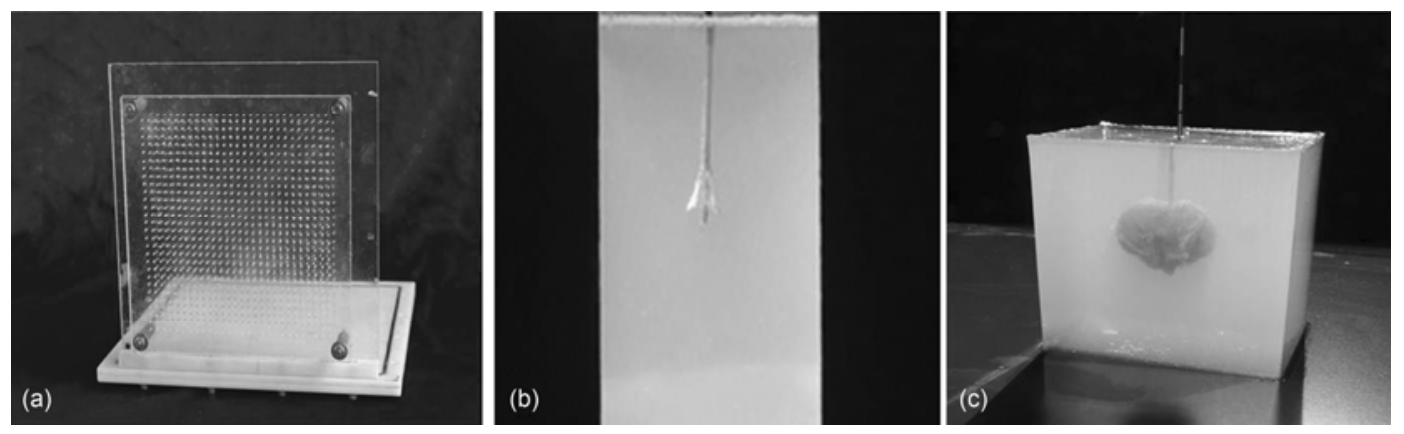

Figure 1 Experimental model. (a) Picture of the double layer organic glass measuring platform; (b) in-vitro phantom with SDE multi-pole radiofrequency electrode inserted; (c) in-vitro prostate phantom with SDE multi-pole radiofrequency electrode inserted. 
points in the maximum $x$ - $z$ plane with $y$ equivalent to 0 .

The RITA1500 RF ablatograph (Mountain View, CA, USA) was used for RF ablation. The plate electrode was pasted onto the base of a special organic glass platform, and the phantom was placed on the measuring platform, with one side closely attached to the front surface of the platform. The SDE multi-pole RF electrode (Angio Dynamics, Manchester, GA, USA) was inserted into the phantom or in-vitro prostate tissue through the original needle channel. After the guide line was connected, we ensure the whole RF instrument composed a complete closed path. We set the RF parameters according to the maximum power of $100 \mathrm{~W}$, average temperature model, the maximum temperature limited to $80^{\circ} \mathrm{C}$, the electrode expansion for $10 \mathrm{~mm}$, and the total time of RF of $11.5 \mathrm{~min}$.

When measuring the temperature, we inserted the thermometric needle into the phantom through a self-made double-layer of organic glass, with each batch of thermometric needle inserted into eight different space points. The insertion direction of the needle should be vertical against the RF electrode. Before every measurement, each thermometric needle was placed in an ice water mixture and warm water of 50 to $70^{\circ} \mathrm{C}$ to correct the measured value of temperature against a mercury thermometer. The real-time temperature change of the whole process was recorded through eight batches of thermometric needles. The temperature change of each space point was measured three times. Following this, the temperature data analyzed with Matlab7.0. First, we equalized the temperature results of the same space point, and then rebuilt the temperature field distribution. In addition, we made comparisons between the two groups of the temperature-time changing curves of the four space points with both $y$ and $z$ equivalent to 0 , and $x$ equivalent to $0,0.25,0.5$, and 0.75 .

After the accomplishment of in-vitro ablation and temperature measurement, we longitudinally cut open the invitro prostate to directly observe the result of RF ablation and make a comparison against the temperature field distribution drawings.

Results were analyzed using SPSS14.0. Discrepancies between variables were tested using the analysis of variance or Wilcoxon signed rank sum test. $P<0.05$ refers to the discrepancy having significance; $P<0.01$ refers to the discrepancy having a high degree of significance; $P>0.05$ refers to the discrepancy having no significance.

\section{Results}

All of the finished phantoms of the in-vitro phantom group showed a faint yellow transparent solid. The ablation lesion of a shape similar to an ellipse can be clearly seen after RF ablation. A white coagulative lesion with a size of about $12 \mathrm{~mm}$ can also be seen in the prostate after RF ablation being applied to the in-vitro prostate group. The two groups were similar in shape (Figure 2).

Temperature measurement points of the in-vitro phantom group during the RF tended to show a zone whose temperature rose faster at $\{(Z, X): 0 \leqslant Z \leqslant 1 \mathrm{~cm}, 0 \leqslant X \leqslant 0.5 \mathrm{~cm}\}$, namely, the temperature rises faster in the zone $1 \mathrm{~cm}$ from the RF needle point to the body, and $0.5 \mathrm{~cm}$ around the needle (Figure 3).

The time-varying results of the ablation zone in the $x-z$ plane with $y$ equivalent to 0 show that boundary areas of the two groups are closer when the temperature is between 50 and $60^{\circ} \mathrm{C}$. However, when the temperature is $70^{\circ} \mathrm{C}$ in the high-temperature region, the discrepancy between the two groups is relatively large (Table 1 ). The temperature measurement outline curve in the $x-z$ plane can also demonstrate that the general shapes of the two groups are analogous at a temperature of 50 and $60^{\circ} \mathrm{C}$. However, at the temperature of $70^{\circ} \mathrm{C}$ and in the high-temperature regions above that are mainly concentrated around the electrode area in the in-vitro prostate group, while it is relatively well-distributed in the in-vitro phantom group (Figures 4 and 5). Finally, a 3D temperature surface model of the in-vitro phantom group, which is established according to the $3 \mathrm{D}$ temperature fields of different times, further proves that the 3D temperature outline shape at $60^{\circ} \mathrm{C}$ is more consistent with the actual effect of the in-vitro prostate tissue after RF ablation (Figure 6).
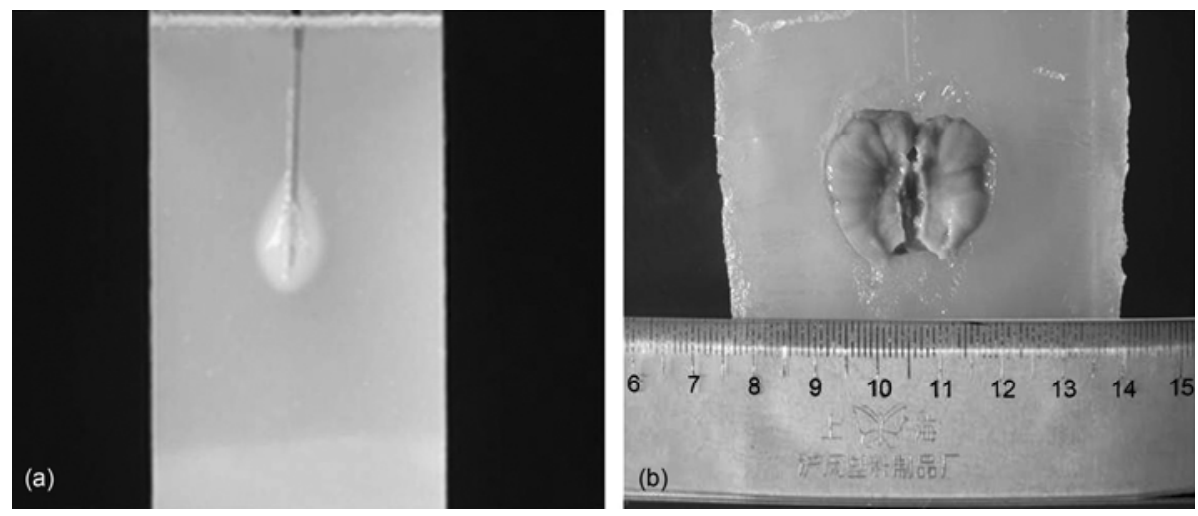

Figure 2 Picture visualizing the in-vitro radio frequency ablation effect of the two experimental groups. (a) In-vitro phantom group; (b) in-vitro prostate group. 

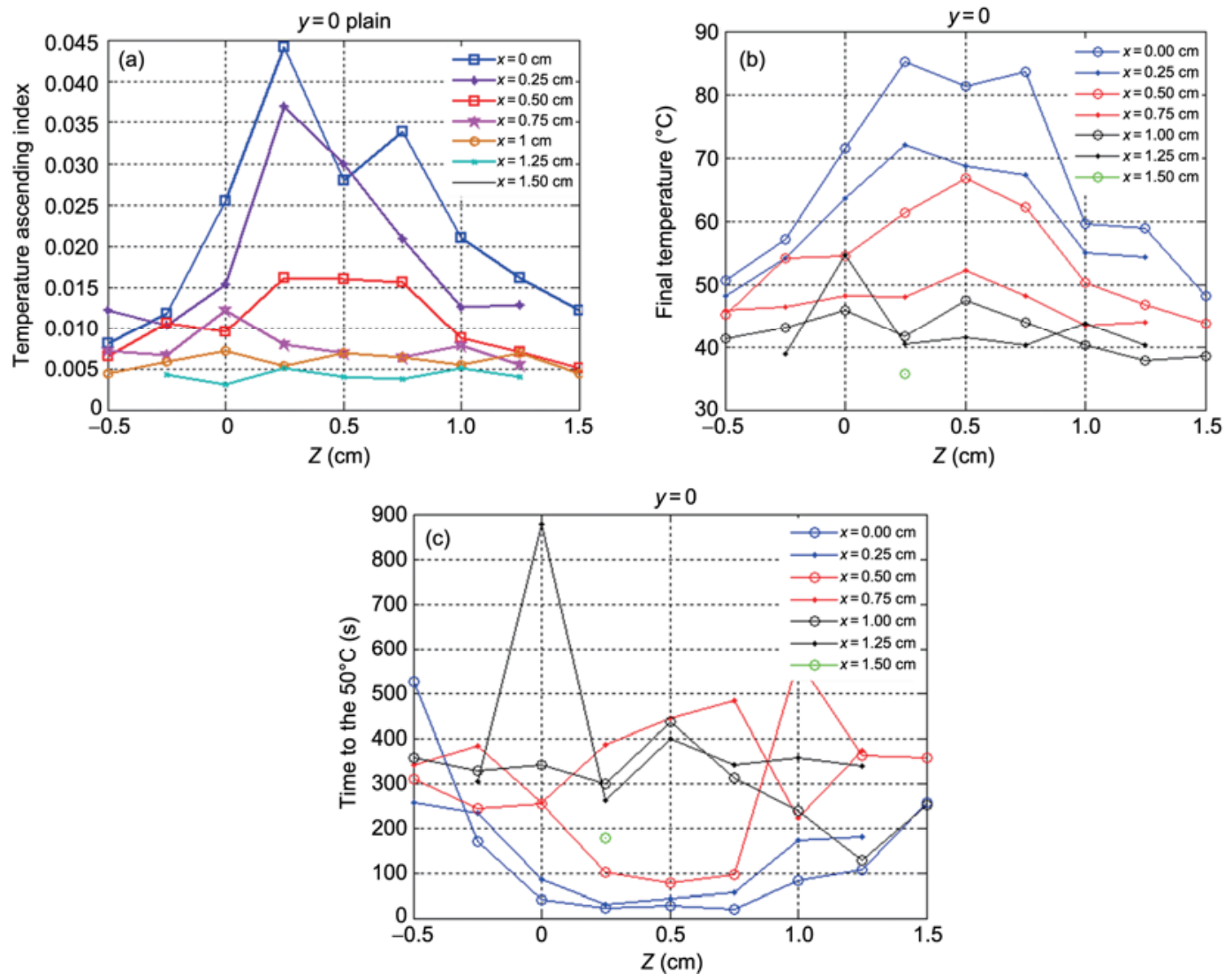

Figure 3 Relationships between temperature changes and measurement points. (a) Relationship between the rising rate of temperature and position; (b) relationship between final temperature (maximum value) and position; (c) relationship between time and position when temperature reached $50^{\circ} \mathrm{C}$.

Table 1 Comparison of the boundary areas at different temperatures of the in-vitro phantom group and in-vitro prostate phantom group during the process of radiofrequency ablation

\begin{tabular}{llllllllllll}
\hline & Time (s) & 60 & 120 & 180 & 240 & 300 & 360 & 420 & 480 & 540 & 600 \\
\hline Boundary area at $50^{\circ} \mathrm{C}\left(\mathrm{cm}^{2}\right)$ & Phantom group & 0.36 & 0.86 & 0.95 & 1.01 & 1.07 & 1.13 & 1.18 & 1.23 & 1.27 & 1.30 \\
& Prostate group & 0.27 & 0.63 & 0.73 & 0.85 & 0.94 & 1.00 & 1.05 & 1.09 & 1.14 & 1.17 \\
Boundary area at $60^{\circ} \mathrm{C}\left(\mathrm{cm}^{2}\right)$ & Phantom group & 0.29 & 0.31 & 0.35 & 0.39 & 0.51 & 0.57 & 0.60 & 0.62 & 0.63 & 0.63 \\
& Prostate group & 0.046 & 0.35 & 0.38 & 0.41 & 0.44 & 0.48 & 0.48 & 0.51 & 0.52 & 0.54 \\
Boundary area at $70^{\circ} \mathrm{C}\left(\mathrm{cm}^{2}\right)$ & Phantom group & 0.16 & 0.39 & 0.39 & 0.40 & 0.40 & 0.40 & 0.40 & 0.40 & 0.42 & 0.44 \\
& Prostate group & 0 & 0.17 & 0.14 & 0.14 & 0.14 & 0.15 & 0.15 & 0.16 & 0.16 & 0.17 \\
\hline
\end{tabular}

There was a skewed distribution of data in the comparison of temperature-time changing curves of the in-vitro phantom group and the in-vitro prostate phantom group at the four space points with $y$ equivalent to $0, z$ equivalent to 0.5 , and $x$ equivalent to $0,0.25,0.5,0.75$. As a result, there exists a significant discrepancy (Sign Rank test $=25638.2, P=$ 0.0001 ) between the two groups during the changing process, however the final temperatures of the two groups showed no significant statistical difference $(P>0.05)$ (Figure 7$)$.

\section{Discussion}

Carcinoma of the prostate is the third most common neo- plastic disease in the world, and the second largest cause of death in American males. Through the assessment of epidemiology, the incidence of the disease in China and some other Asian countries is lower than in America. However, with the normalizing application of prostate specific antigen testing in these developing countries, the detection rate of prostate carcinoma has increased from $16 \%$ to $118 \%$, and it is inferred that the disease incidence of prostate carcinoma (mainly early prostate carcinoma) is likely to increase as well [7].

Currently the first choice of treatment modality is radical prostatectomy. Although the effectiveness of surgery is well-recognized, there exists the potential for certain complications and associated mortality rate. However, for those 

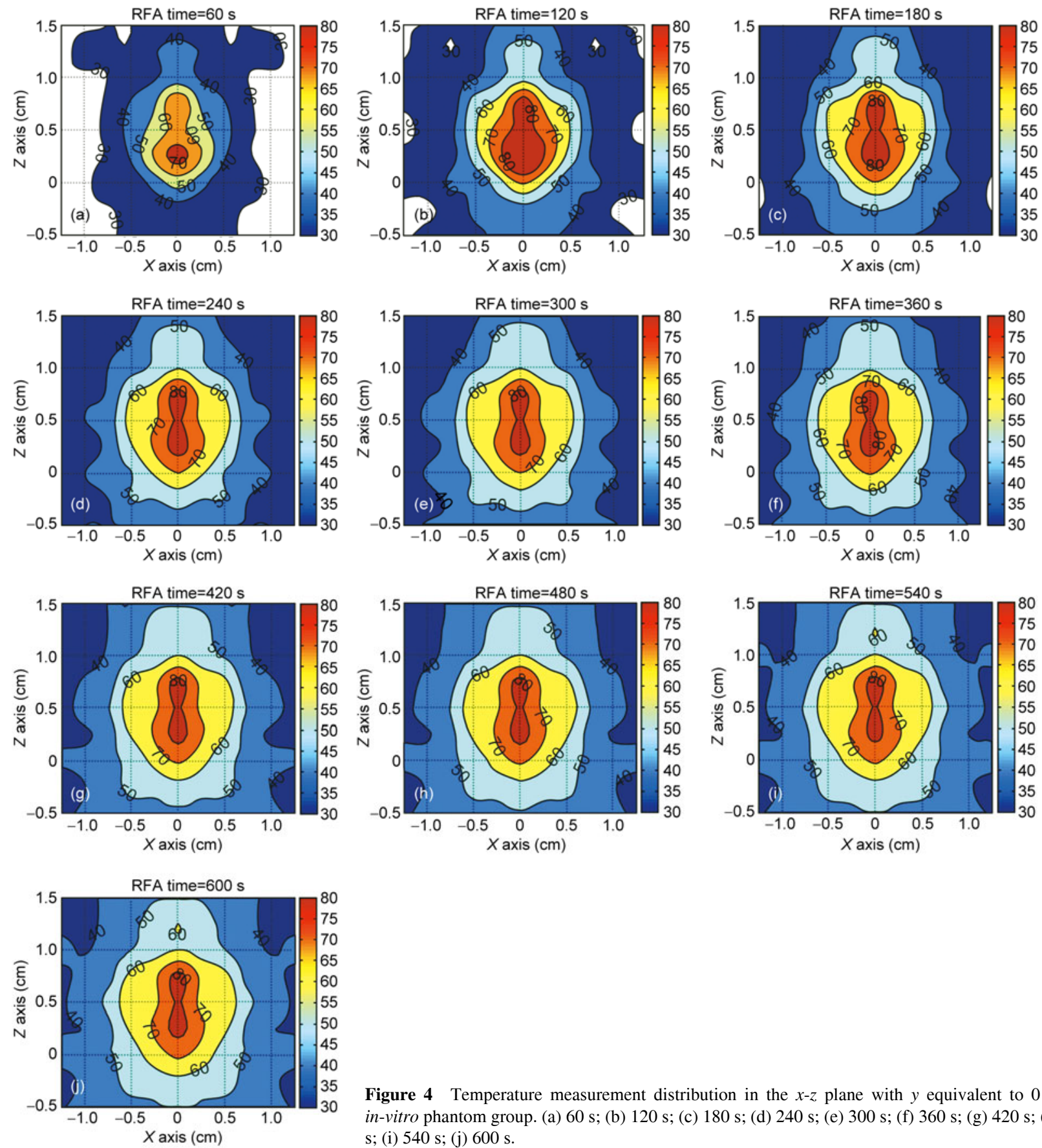

Figure 4 Temperature measurement distribution in the $x$-z plane with $y$ equivalent to 0 of the in-vitro phantom group. (a) $60 \mathrm{~s}$; (b) $120 \mathrm{~s}$; (c) $180 \mathrm{~s}$; (d) $240 \mathrm{~s}$; (e) $300 \mathrm{~s}$; (f) $360 \mathrm{~s}$; (g) $420 \mathrm{~s}$; (h) 480 s; (i) $540 \mathrm{~s}$; (j) $600 \mathrm{~s}$.

patients who have surgical contraindications and cannot bear the surgery, a minimally invasive treatment would undoubtedly be a better option. One such treatment technology is RF ablation. With this kind of treatment, many patients could receive treatment in an outpatient clinic. The treatment time is short, and only a few antibiotics and pain relief treatment are needed after the treatment. There are no known major complications, and the dwelling time of the urethral catheter is significantly reduced. As a result, it is favored by patients with prostate carcinoma. However, the curative effect when conducting treatment is not stable be- cause the ablation effect is influenced by the surrounding anatomical structures, the size of the tumor, pathological biopsy results, images during the operation and other elements [8]. Only by accurate parameterized control over the $\mathrm{RF}$ ablation lesion and relevant parameters is it possible to further stabilize and enhance the curative effect of RF ablation. However, a profound understanding of the temperature field distribution would be the basis for an enhanced and reproducible curative effect and enable parameterized treatment design. Currently, the relevant literature is focused mainly at the stage of experimentation on animals [9], 

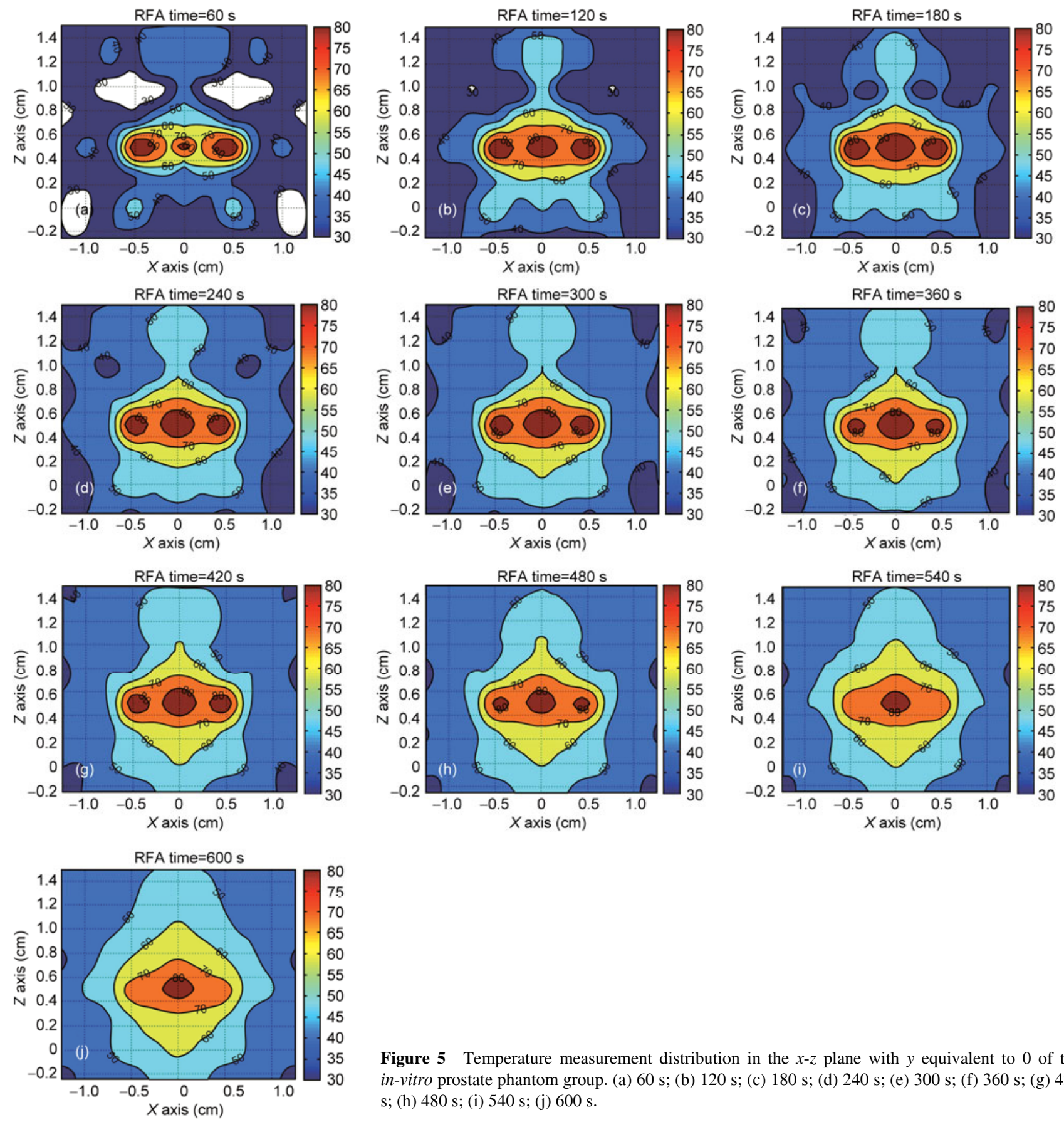

Figure 5 Temperature measurement distribution in the $x-z$ plane with $y$ equivalent to 0 of the in-vitro prostate phantom group. (a) $60 \mathrm{~s}$; (b) $120 \mathrm{~s}$; (c) $180 \mathrm{~s}$; (d) $240 \mathrm{~s}$; (e) $300 \mathrm{~s}$; (f) $360 \mathrm{~s}$; (g) 420 s; (h) $480 \mathrm{~s}$; (i) $540 \mathrm{~s}$; (j) $600 \mathrm{~s}$.

and in-vitro visualization phantoms have not been established to simulate the RF ablation process of in-vivo prostates and relevant temperature field distribution. Such a phantom would enable the simplification of experimental procedures and serve as a guide for clinical treatments. Consequently, this was the aim of this experiment.

The raw materials used for biological thermal destruction phantoms mainly consist of agar, sodium carboxy methyl cellulose and tissue homogenate. However, there always exist imperfections in the complex preparation technology, large inaccuracies in measurement and a low degree of vis- ualization. In this work, we adopted acrylamide as a raw material, and the phantom was made by simple preparation technologies to be transparent and flexible. The ablation lesion inside the phantom after the application of RF could subsequently be observed by the naked eye. We measured the thermodynamic parameters of the phantom, which were close to those of canine and human prostate tissues (Table 2). Therefore, these results are completely applicable to the simulation of canine and human prostate tissues in thermodynamic tests [10]. In addition, we adopted egg white as a visualization reagent, whose solidification point is between 
(a)

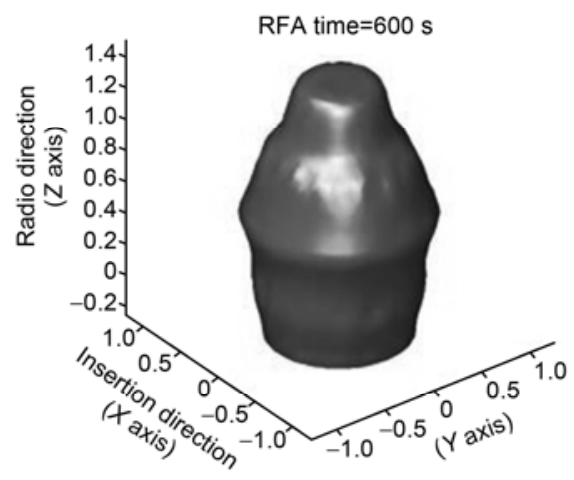

(b)

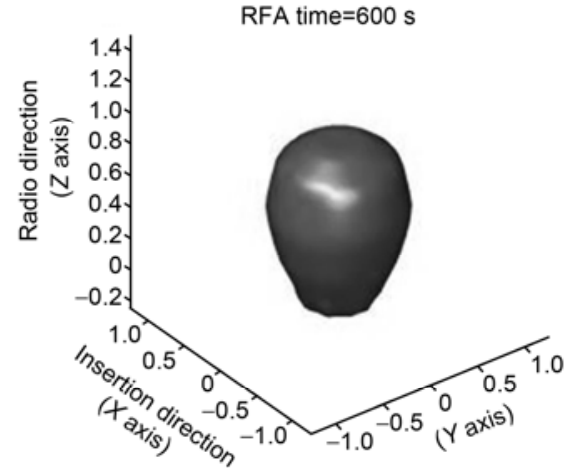

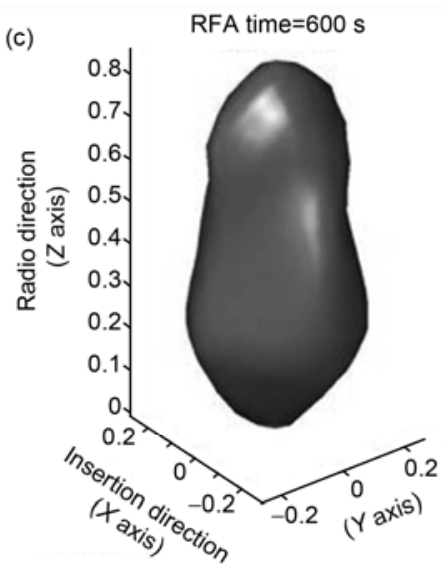

Figure 6 Reconstructed diagram of the three dimensional temperature surface model of the in-vitro phantom group. (a) Three dimensional temperature surface model at $50^{\circ} \mathrm{C}$; (b) three dimensional temperature surface model at $60^{\circ} \mathrm{C}$; (c) three dimensional temperature surface model at $70^{\circ} \mathrm{C}$.

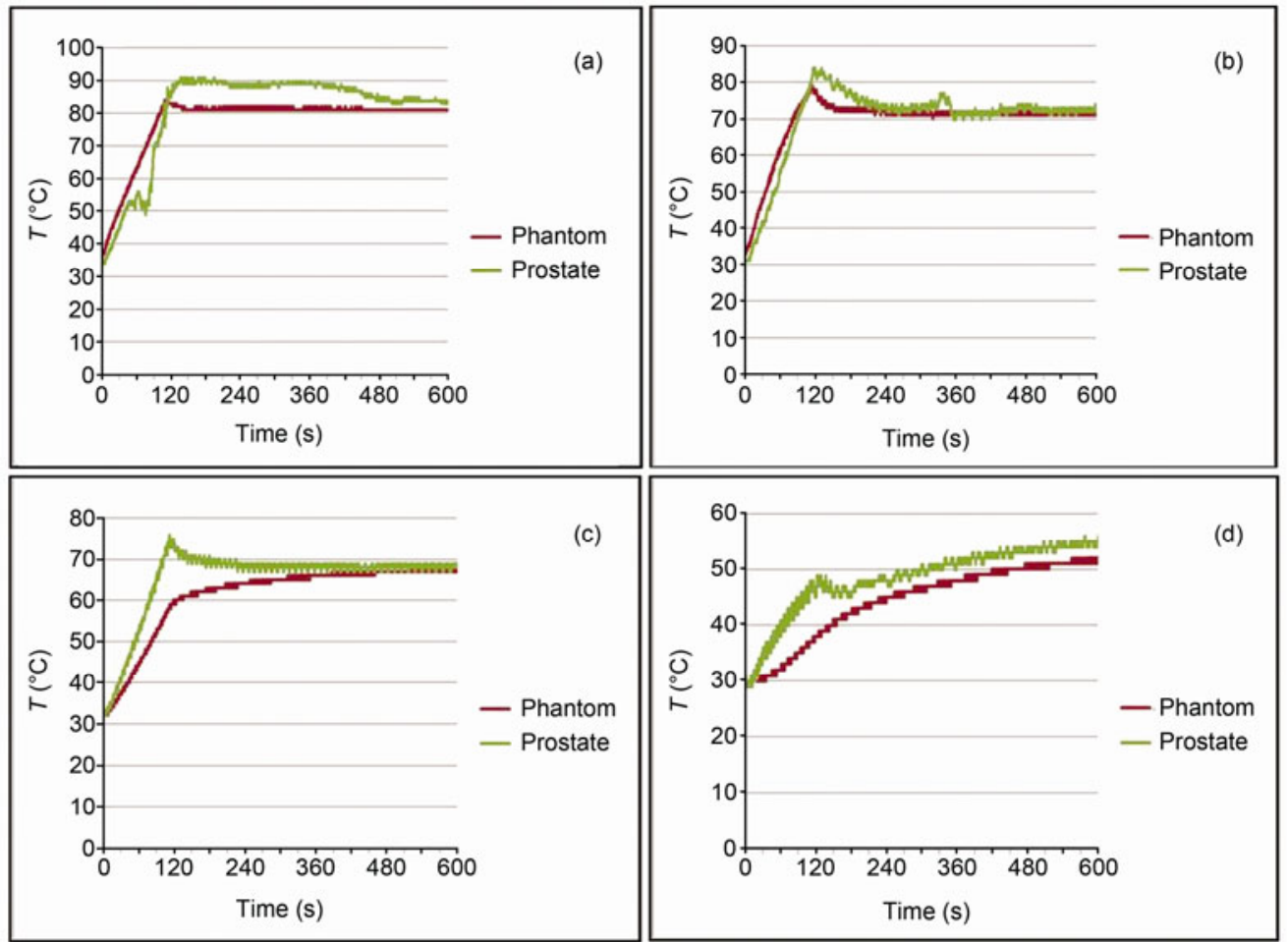

Figure 7 Temperature-time change comparison between the in-vitro phantom group and the in-vitro prostate phantom group at the same space points. (a) Comparison of the two groups at $Y=0, Z=0.5, X=0$; (b) comparison of the two groups at $Y=0, Z=0.5, X=0.25$; (c) comparison of the two groups at $Y=0, Z=0.5$, $X=0.5$; (d) comparison of the two groups at $Y=0, Z=0.5, X=0.75$. 
Table 2 Thermodynamic parameters of the visualization phantom with egg white at a volume concentration of $20 \%$

\begin{tabular}{lc}
\hline \multicolumn{1}{c}{ Thermodynamic parameters } & Temperature $\left(25^{\circ} \mathrm{C}\right)$ \\
\hline Coefficient of heat transfer $\left(\mathrm{W} \mathrm{m}^{-1}{ }^{\circ} \mathrm{C}^{-1}\right)$ & $0.389 \pm 0.044$ \\
Density $\rho\left(\mathrm{g} \mathrm{mL}^{-1}\right)$ & 1.016 \\
Specific heat Cpw $\left(\mathrm{J} \mathrm{kg}^{-1}{ }^{\circ} \mathrm{C}^{-1}\right)$ & 3611.55 \\
Coefficient of heat transfer $\lambda\left(\mathrm{W} \mathrm{m}^{-1} \mathrm{~K}^{-1}\right)$ & 0.52 \\
\hline
\end{tabular}

60 and $70^{\circ} \mathrm{C}$. This is closer to the required temperature of the curative effect in the process of $\mathrm{RF}$ ablation against prostate tissue. Meanwhile its performance price ratio is higher than if using calf serum as the visualization reagent, as is conventionally done $[11,12]$.

Because the interval of every measurement point was $2.5 \mathrm{~mm}$, we obtained as much temperature change data as possible which was sufficient for a $2 \mathrm{D}$ or $3 \mathrm{D}$ temperature distribution to be simulated. Additionally, we measured the temperature change in each space point three times so that the bias due to the measurement could be minimized.

In the comparison of the heat distribution area of the ablation lesions, we mainly conducted a comparative analysis of the $x-z$ plane with $y$ equivalent to 0 for the two groups, because this plane is where the largest ablation area is situated. The analysis of the temperature field of the plane is thus likely to have the highest representation of the overall distribution. Our results show that the outlines of temperature distribution of the two groups are closer at 50 and $60^{\circ} \mathrm{C}$, but differ by a large margin at $70^{\circ} \mathrm{C}$. In the in-vitro prostate phantom group, the high temperature zone around the electrode is apparent, which was not observed in the other group. We considered the prostate tissue to be mainly glandular tissue with high moisture content. As the urethra passes through it, the structure is relatively complicated. During the application of RF, the high moisture content means that ion components around the electrode are greater, which results in the rapid formation of a solidification lesion around the electrode. As a result, we are able to observe the high temperature zone around the electrode. With increasing time, the moisture content in the tissue decreases. When the electrode's self-installed temperature measurement thermocouple temperature reaches the target temperature, the instrument then automatically reduces the RF power so that the temperature around the electrode decreases by a small amount. The moisture content in the phantom is composed of acrylamide, and the moisture in the phantom group is less than that found in prostate tissue, which may have led to the observed results. In addition, the well-distributed modular organization and relatively smaller expanding diameter of the electrode lead to the formation of a uniform distribution of the electric field of the RF between the three electrodes.

It has been well established that the cell may be damaged when the temperature reaches $45^{\circ} \mathrm{C}$ [13], and die at $50^{\circ} \mathrm{C}$ [14]. However, some studies have suggested that the cell will die when the temperature reaches $54^{\circ} \mathrm{C}$ and is sustained for $1 \mathrm{~min}$ or reaches $60^{\circ} \mathrm{C}$ [15]. Although the outlines of the two groups at 50 and $60^{\circ} \mathrm{C}$ were similar, we consider the outline result of the in-vitro phantom group at $60^{\circ} \mathrm{C}$ to roughly simulate the RF ablation result of the in-vitro prostate according to data from the previously mentioned study [13-15]. The in-vitro phantom group's reconstructed diagrams of the 3D temperature surface model also show that the $3 \mathrm{D}$ temperature surface model at $60^{\circ} \mathrm{C}$ is closer to the visual appearance of the RF ablation lesion from the in-vitro phantom or in-vitro prostate groups.

In a further analysis of the temperature change of the same four space points, the RF applied to the in-vitro prostate can produce the target temperature sooner than in the in-vitro phantom. The reason for this may be related to the thermal characteristics of the prostate tissue or the moisture content of the tissue. However, because the energy of the $\mathrm{RF}$ is sufficiently large, the final temperatures of the two groups have no obvious distinctions. The results from the in-vitro phantom group and in-vitro prostate group are relatively consistent and further demonstrate that the results of the RF temperature field can roughly reflect those of the actual treatment.

There are some limitations to this research. For example, the space points used in the comparison of the temperature-time change of the two groups are not very large in number, and may not accurately reflect the overall change in the temperature field. The design will be optimized in future research. Furthermore, the number of adult dogs available limited the simulation of the temperature field distribution in prostate tissue. Because temperature changes could only be obtained in one plane in each sample, more samples will be needed order to obtain the temperature distribution in a different plane. Another limitation is that the presence of the temperature measurement needle may interfere with the electric-field of the RF. Although the needle was painted with an insulating layer, it still acts as an electric conductor, so an influence can still exist. However, the extent of the problem requires further research.

In conclusion, these experiments show that RF ablation appearance and temperature field distribution is consistent with that of in-vitro biological tissue, and can be obtained during RF ablation by taking advantage of a visualization phantom made of acrylamide. The outline shape of the isothermal at $60^{\circ} \mathrm{C}$ and the reconstructed diagram of the 3D surface temperature are similar to those of the actual ablation lesion. Therefore, we consider the 3D temperature field change obtained in the in-vitro ablation model to have instructive value to the understanding of temperature change from RF ablation of in-vivo tissue, and can serve as a significant reference to follow-up clinical research and experimental work.

1 Bonillo G M, Gimeno A V, Jiménez J F. Radiofrequency interstitial tumor ablation (RITA) for the treatment of localised prostate cancer. 
Actas Urol Esp, 2007, 31: 627-632

2 Djavan B, Zlotta A R, Susani M, et al. Transperineal radiofrequency interstitial tumor ablation of the prostate: Correlation of magnetic resonance imaging with histopathologic examination. Urology, 1997, 50: 986-992

3 Zlotta A R, Djavan B, Matos C, et al. Percutaneous transperineal radiofrequency ablation of prostate tumour: Safety, feasibility and pathological effects on human prostate cancer. Br J Urol, 1998, 81: 265-275

4 McGahan J P, Griffey S M, Budenz R W. Percutaneous ultrasound-guided radiofrequency electrocautery ablation of prostate tissue in dogs. Acad Radiol, 1995, 2: 61-65

5 Bhowmick S, Swanlund D J, Coad J E, et al. Evaluation of thermal therapy in a prostate cancer model using a wet electrode radiofrequency probe. Endourol, 2001, 15: 629-640

6 Chang I A. Considerations for thermal injury analysis for RF ablation devices. Open Biomed Eng J, 2010, 4: 3-12

7 Raymundo E M, Rice K R, Chen Y, et al. Prostate cancer in Asian Americans: Incidence, management and outcomes in an equal access healthcare system. BJU Int, 2010, 29, Doi: 10.1111/j.1464-410X. 2010.09685.x

8 Shariat S F, Raptidis G, Masatoschi M, et al. Pilot study of radiofrequency interstitial tumor ablation (RITA) for the treatment of ra- dio-recurrent prostate cancer. Prostate, 2005, 65: 260-267

9 Zhu L, Pang L, Xu L X. Simultaneous measurements of local tissue temperature and blood perfusion rate in the canine prostate during radio frequency thermal therapy. Biomech Model Mechanobiol, 2005, 4: $1-9$

10 Zhu L, Xu L X, Chencinski N. Quantification of the 3-D electromagnetic power absorption rate in tissue during transurethral prostatic microwave thermotherapy using heat transfer model. IEEE Trans Biomed Eng, 1998, 45: 1163-1172

11 Kenjin T, Yukio K, Toshiaka W, et al. Polyacrylamide gel containing egg white as new model for irradiation experiments using focused ultrasound. Ultrasound Med Biol, 2004, 30: 1419-1422

12 Song J W, Xu M T, Cheng L. Theoretical analysis of a method for segmented heat exchanger design. Chinese Sci Bull, 2011, 56: 21792184

13 Hearse D J, Yellon D M. Therapeutic Approaches to Myocardial Infarct Size Limitation. New York: Raven Press, 1984. 163-184

14 Haemmerich D, Laeseke P F. Thermal tumour ablation: Devices, clinical applications and future directions. Int J Hyperthermia, 2005, 21: 755-760

15 Dong B W, Liang P, Yu X L, et al. Sonographically guided microwave coagulation treatment of liver cancer: An experiment and clinical study. AJR Am J Roentgenol, 1998, 171: 449-454

Open Access This article is distributed under the terms of the Creative Commons Attribution License which permits any use, distribution, and reproduction in any medium, provided the original author(s) and source are credited. 